\title{
Statistical Methods for Image Segmentation and Tomography Reconstruction
}

\author{
M.L. Comer*, C.A. Bouman*, and J.P. Simmons** \\ *School of Electrical and Computer Engineering, Purdue University, West Lafayette, IN 47907 \\ **Materials and Manufacturing Directorate, Air Force Research Laboratory AFRL/RXLMD, \\ Dayton, $\mathrm{OH}$
}

We describe statistical methods for two image processing problems that are of interest in materials science. The first application is image segmentation. To segment an image means, roughly speaking, to partition the image into several regions in such a way that certain image characteristics of interest are homogeneous within each region and heterogeneous between regions. The second application we will discuss is tomography reconstruction.

We will discuss a general framework for statistical methods for image processing, and then decribe the usage of our statistical methods for image segmentation and tomography reconstruction for materials science and medical image analysis. The statistical approach defines models for the distributions of image data, and seeks solutions to image processing problems by minimizing a suitably defined cost function based on these image models. For the methods we will discuss, we define image models for both the observed image data and for the solution image data for the problem of interest. These models allow us to incorporate prior information that can dramatically improve the performance of the methods.

For the image segmentation problem, we have developed a method known as the "expectationmaximization/maximization of the posterior marginals" (EM/MPM) algorithm, which addresses the problem of classifying pixels in an image into different texture classes [1,2,3]. The individual pixel classifications, or labels, form a multidimensional field, with the same spatial resolution as the observed image, in which the value at a given spatial location reflects the texture to which the corresponding pixel in the observed image belongs. This field containing the individual pixel classifications is referred to as the label field. The label field is unknown and must be estimated from the observed image. We will describe one of the most popular statistical image models, the Markov random field (MRF) model, which we use for the label field in the EM/MPM method. We will present results showing the capabilities of the EM/MPM method. (See Figure 1 for examples.)

For tomographic reconstruction, statistical methods based on the MRF model have proven to be very effective [4,5]. We have developed methods that pose the reconstruction problem as a statistical optimization problem. We will discuss the statistical models we use for tomography data and for the optimal reconstruction from that data, as well as optimization procedures that approximate this optimal reconstruction.

Our basic tomography reconstruction approach operates at a single, fixed scale. We have demonstrated that for tomographic reconstruction, a multiscale processing approach is advantageous [5]. Our multiscale method works by first reconstructing a coarse resolution reconstruction, then progressing from the coarse scale to finer scales, and finally obtaining a reconstruction at the highest resolution feasible given the tomography data available. We will describe this multiscale extension 
of our basic fixed-scale reconstruction method, and show results comparing the performance of this approach to other methods.

\section{References}

[1] M. L. Comer and E. J. Delp, "The EM/MPM Algorithm for Segmentation of Textured Images: Analysis and Further Experimental Results," IEEE Transactions on Image Processing, Vol. 9, No. 10, October 2000, pp. 1731-1744.

[2] J. P. Simmons, P. Chuang, M. Comer, J. E. Spowart, M. D. Uchic, and M. De Graef, "Application and Further Development of Advanced Image Processing Algorithms for Automated Analysis of Serial Section Image Data," Modelling and Simulation in Materials Science and Engineering, Vol. 17, No. 2, March 2009.

[3] J.P. Simmons, B. Bartha, M. De Graef, and M. Comer, "Development of Bayesian Segmentation Techniques for Automated Segmentation of Ttianium Alloy Images," Microscopy and Microanalysis, vol. 14 (S2), pp. 602-603, 2008.

[4] S. Geman and D. McLure, "Statistical Methods for Tomographic Image Reconstruction," Bull Int Stat Inst LII-4, 5-21, 1987.

[5] T. Frese, C.A. Bouman, and K. Sauer, "Multiscale Bayesian Methods for Discrete Tomography," Discrete Tomography: Foundations, Algorithms and Applications, edited by Gabor T. Herman and Attila Kuba, pp. 237-261, Birkhauser Boston, Cambridge, MA, 1999.

[6] We would like to acknowledge Hamish Fraser of the Ohio State University and Mike Uchic of AFRL for providing image data.
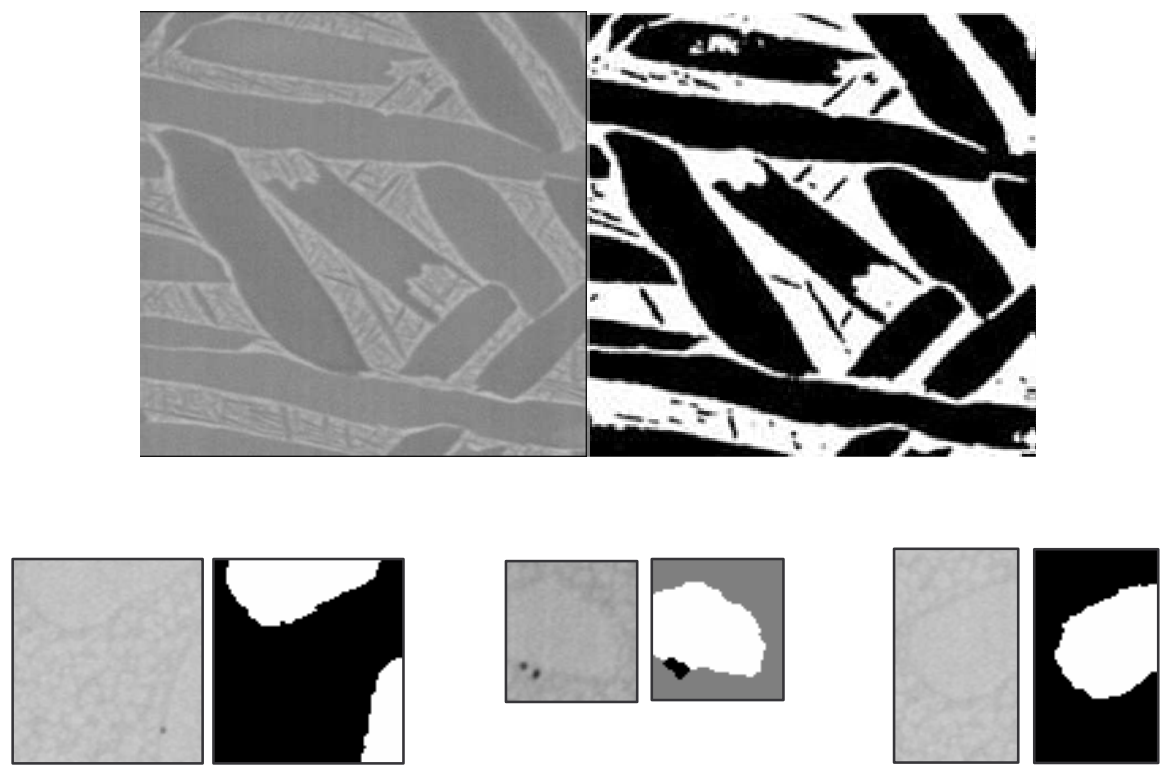

FIG. 1. Examples of materials images (top: Ti alloy; bottom: Ni alloy grains) and segmentations obtained using EM/MPM. 